\title{
M-Polynomials and Topological Indices for Line Graphs of Chain Silicate Network and H-Naphtalenic Nanotubes
}

\author{
Muhammad Irfan $\mathbb{D}^{1},{ }^{1}$ Hamood Ur Rehman, ${ }^{1}$ Hassan Almusawa $\mathbb{D}^{\circ},{ }^{2}$ Saffina Rasheed, \\ and Imran Abbas Baloch $\mathbb{D I}^{3,4}$ \\ ${ }^{1}$ Department of Mathematics, University of Okara, Okara, Pakistan \\ ${ }^{2}$ Department of Mathematics, College of Sciences, Jazan University, Jazan 45142, Saudi Arabia \\ ${ }^{3}$ Abdus Salam School of Mathematical Sciences, Government College University, Lahore, Pakistan \\ ${ }^{4}$ Higher Education Department, Government College for Boys Gulberg, Lahore, Punjab, Pakistan \\ Correspondence should be addressed to Imran Abbas Baloch; iabbasbaloch@gmail.com
}

Received 5 January 2021; Revised 24 February 2021; Accepted 6 March 2021; Published 20 March 2021

Academic Editor: Ahmet Sinan Cevik

Copyright (C) 2021 Muhammad Irfan et al. This is an open access article distributed under the Creative Commons Attribution License, which permits unrestricted use, distribution, and reproduction in any medium, provided the original work is properly cited.

\begin{abstract}
Graph theory has provided a very useful tool, called topological index, which is a number from the graph $M$ with the property that every graph $N$ isomorphic to $M$ value of a topological index must be same for both $M$ and $N$. Topological index is a descriptor in graph theory which is used to quantify the physio-chemical properties of the chemical graph. In this paper, we computed closed forms of M-polynomials for line graphs of $\mathrm{H}$-naphtalenic nanotubes and chain silicate network. From M-polynomial, we obtained some topological indices based on degrees.
\end{abstract}

\section{Introduction}

A graph is an order pair, $M=(V(M), E(M))$, where $V(M)$ is the set of $m$ vertices or points and $E(M)$ is the set of $n$ unordered pairs of distinct vertices of $V(M)$. Every ordered pair of points $\left(r_{i}, s_{j}\right)$ or $(i, j)$ is a line or edge $e_{i, j}$ of $M$ if $(i, j) \in E(M)$.

In a graph $M=(V(M), E(M)),|V|$ represents the total number of vertices called order of $M$ denoted as $m$ while $|E|$ represents the total number of lines or edges called size of $M$ denoted as $n$. Fundamental theorem of graph theory is stated as the sum of all the degrees of vertices in a graph $M$ which is equal to two times the total edges in a graph. Mathematically, it can be represented as

$$
\sum_{i=1}^{n} \operatorname{deg}\left(r_{i}\right)=2|E| \text {. }
$$

For other undefined notations, see [1-3], a label graph whose vertices and edges correspond to the atoms and chemical bonds of the compound, respectively, this type of graph is said to be a chemical graph. Its vertices and edges are labeled with the kinds of the corresponding atoms and types of bonds. The numerical parameters of a graph which describe its topology are said to be the topological indices or graph invariants. The simplest topological indices do not recognize double bonds and types of atoms $(C, N, O$, etc.) and ignore hydrogen atoms. The topological indices are defined for undirected connected molecular graphs only.

The topological indices of the chemical or molecular graphs also help us to investigate the physio-chemical properties and boiling activities, i.e., heat of formation, surface area, surface tension, vapors pressure, and boiling points of the involved molecular or chemical compound, see $[4,5]$. Cheminformatics is another emerging field in which quantitative structure property relationship (QSPR) and quantitative structure activity relationship (QSAR) predict the biological activities' properties of the nanomaterials. The study of topological indices is used in (QSPR) and (QSAR) [2]. In graph theory, there are various types of topological indices such as counting-related indices, based on distance and based on degrees. The most studied topological indices 
are topological indices based on degrees. These indices are most important and have many useful applications. In this paper, we discussed M-polynomial and some degree-based topological indices. M-Polynomial is rich in producing source of many topological indices based on degrees which correspond to chemical properties of the material under investigation.

\section{Basic Definitions and Literature Review}

In this section, we study some basic definitions and literature review. Let a graph $M$ and its line graph $L(M)$ be a graph such that each vertex of $L(M)$ represents an edge of $M$ and two vertices of $L(M)$ are connected if their respective edges share a common vertex. The terminologies and notations of the graphs which are used in this paper are standard. In this paper, we discussed topological indices based on degrees. In 1972, Trinajstic et al. [6] defined a pair of topological indices based on degrees which are first and Zagreb second indices for $\Pi-$ energy of conjugated molecules. For more detail about topological indices, see [7-9]. Let $M=(V, E)$ be a molecular graph with $d(k)$ and $d(l)$ which are degrees of vertices $k$ and $l$, and then the first and second Zagreb indices are represented by $M_{1}(M)$ and $M_{2}(M)$, respectively, and stated as

$$
\begin{aligned}
& M_{1}(M)=\sum_{k l \in E(M)}(d(k)+d(l)), \\
& M_{2}(M)=\sum_{k l \in E(M)}(d(k) d(l)) .
\end{aligned}
$$

After these indices, a new form of second Zagreb index was introduced by Nikolic et al. [6] which is known as modified second Zagreb index. Modified second Zagreb index is another degree-based topological index denoted by $M M_{2}(M)$ and defined as

$$
M M_{2}(M)=\sum_{k l \in E(M)}\left(\frac{1}{d(k) d(l)}\right) .
$$

Third Zagreb index was first used in 2011 by Fath-Tabar [2], denoted by $M_{3}(M)$ and defined as

$$
M_{3}(M)=\sum_{k l \in E(M)}(|d(k)-d(l)|) .
$$

This topological index which computed surface area of polychlorobiphenyls is called symmetric division index [3] and defined as

$$
\operatorname{SDD}(M)=\sum_{k l \in E(M)}\left(\frac{\max (d(k), d(l))}{\min (d(k), d(l))}+\frac{\min (d(k), d(l))}{\max (d(k), d(l))}\right) .
$$

In 2010, augmented Zagreb index was introduced by Furtula et al. [3] which is stated as follows:

$$
\operatorname{AZI}(M)=\sum_{k l \in E(M)}\left(\frac{d(k) d(l)}{d(k)+d(l)-2}\right)^{3} .
$$

This remarkable predictive index is used to compute the heat of formation in heptane and octanes.

In 2009, Trinajstic and Zhou derived a index, called sum connectivity index. This index $(S(M))$ is stated as

$$
S(M)=\sum_{k l \in E(G)}\left(\frac{1}{\sqrt{d(k)+d(l)}}\right) .
$$

Inverse sum index, is the graph invariant which was studied as a significant descriptor of surface area for isomers of octane and stated as

$$
\operatorname{IS}(M)=\sum_{k l \in E(M)}\left(\frac{d(k) d(l)}{d(k)+d(l)}\right) .
$$

In 1975, Randic [10] introduced the very first and oldest topological index based on degree stated as

$$
R_{-(1 / 2)}(M)=\sum_{k l \in E(M)}\left(\frac{1}{\sqrt{d(k) d(l)}}\right) .
$$

In 1998, Bollobas and Erdos [11] and Amic et al. [12] independently defined general Randic index for a simple connected graph $M$ and for a real number $\alpha$ as

$$
R_{\alpha}(M)=\sum_{k l \in E(M)}(d(k) d(l))^{\alpha} .
$$

In 2005, both $\mathrm{Hu}$ and $\mathrm{Li}$, mathematician and theoretical chemist, have extensively studied it. Caporossi et al. [13] in 2003 have established very interesting and important mathematical properties.

The other harmonic index is defined as

$$
H(M)=\sum_{k l \in E(M)}\left(\frac{2}{d(k)+d(l)}\right) .
$$

Various graph polynomials were introduced in the literature, some of them are Hosoya polynomial, matching polynomial, Zhang-Zhang polynomial, Schultz polynomial, Tutte polynomial, etc. In this section, we study a polynomial called the M-polynomial and show that its role for indices based on degree is similar to the role of the Hosoya polynomial for indices based on distance. See for detail $[1,3,14]$. In 2015, Munir et al. [15] introduced the concept of $\mathrm{M}$-polynomial. The edge of the M-polynomial is the source of knowledge which contains information about degreebased graph invariants. For M-Polynomial of different graphs, see [16-19]. Let $M$ be molecular graph and $m_{p, q}$, where $p, q$ greater than or equal to 1 be the total number of edges of $M$ such that $(d(k), d(l))$ is equal to $p, q$. M-Polynomial of $M$ is stated as

$$
M\left(M, r_{1}, s_{1}\right)=\sum_{p \leq q}\left[m_{p, q}(M)\left(r_{1}\right)^{p}\left(s_{1}\right)^{q}\right] .
$$

Relation of topological indices and M-polynomials is defined as 


$$
\begin{aligned}
D_{r 1} & =\frac{\partial\left(g\left(r_{1}, s_{1}\right)\right)}{\partial r_{1}}, \\
D_{s 1} & =\frac{\partial\left(g\left(r_{1}, s_{1}\right)\right)}{\partial s_{1}}, \\
D_{s 1} \beta D_{r 1} \alpha & =D_{s 1} \beta D_{r 1} \alpha\left(\sum_{p \leq q}\left[m_{p, q}(M) r_{1}^{p} s^{q}\right]\right) \\
& =\sum_{p \leq q}\left[m_{p, q} p \alpha q \beta(M) r_{1}^{p} y_{1}^{s}\right], \\
S_{r 1} & =\int_{0}^{r 1} \frac{g\left(t, s_{1}\right) \mathrm{d} t}{t}, \\
S_{s 1} & =\int_{0}^{s 1} \frac{g\left(r_{1}, t\right) \mathrm{d} t}{t}, \\
J\left(g\left(r_{1}, s_{1}\right)\right) & =g\left(r_{1}, s_{1}\right), \\
Q_{\alpha}\left(g\left(r_{1}, s_{1}\right)\right) & =r_{1}^{\alpha} g\left(r_{1}, s_{1}\right),
\end{aligned}
$$

where $\alpha \neq 0$. To know in details for these operators, see $[5,12]$.

The following lemmas [15] are important for our results.

Lemma 1. Let $M$ be a graph with $k, l \in V(M)$ and $e=k l \in E(M)$. Then,

$$
d(e)=d(k)+d(l)-2 .
$$

Lemma 2. Let $M$ be a graph of size $l$ and order $k$. Then, the line graph $L(M)$ of $M$ is a graph of order $k$ and size $(1 / 2) M_{1}(M)-l$.

Here, we give derivation of all above-discussed indices from $M\left(M, r_{1}, s_{1}\right)$, as shown in Table 1 .

\section{Results and Discussion}

In this section, we discuss construction of line graphs and some topological indices based on degrees of chain silicate network [20] and $\mathrm{H}$-naphtalenic nanotubes [21].

In the first line graph of chain silicate network, silicates are the most complicated, largest, and interesting class of minerals. Tetrahrdron $\left(\mathrm{SiO}_{4}\right)$ are found nearly in all the silicate. We get silicate from metal carbonates with sand or from fusing metal oxides. In chemistry, in $\mathrm{SiO}_{4}$ tetrahedron, the center vertex represents the silicon icon and the corner vertices of $\mathrm{SiO}_{4}$ tetrahedron represent oxygen we get chain silicate network ions. There are many methods to construct the silicate network of the tetrahedron. If tetrahedron silicate joins in a row, then we get chain silicate network, see [20] for details. Chain Silicate CS(1) and its line graphs L(CS(p)) for different values of $\mathrm{p}$ are shown in Figures 1 to $4 . \operatorname{In} L(\mathrm{CS}(p))$ graph, total vertices and total edges are $6 p$ and $21 p-9$, respectively. There are three types of vertices having degrees 4,7 , and 10.

Let $m_{k, l}$ represent total edges connecting the vertices of degrees $d(k)$ and $d(l)$. The line graph of chain silicate network contains only $m_{4,4}, m_{4,7}, m_{7,7}, m_{7,10}$, and $m_{10,10}$ edges. The number of $m_{4,4}, m_{4,7}, m_{7,7}, m_{7,10}$, and $m_{10,10}$ edges in different $p$ values is mentioned in Table 2 . We discuss the results for only $p>3$.

Theorem 1. Let $M=L(C S(p))$ be the edge version of chain silicate network. Then, $M$-polynomial of $M$ is

$$
\begin{aligned}
M(M, r, s)= & 6 r^{4} s^{4}+(4 p+4) r^{4} s^{7}+(8 p-2) r^{7} s^{7} \\
& +(8 p-14) r^{7} s^{10}+(p-3) r^{10} s^{10}
\end{aligned}
$$

Proof

$$
\begin{aligned}
M(M, r, s)= & \sum_{p \leq q}\left[m_{p, q}(M) r^{p} s^{q}\right] \\
= & \sum_{4 \leq 4}\left[m_{4,4}(M) r^{4} s^{4}\right]+\sum_{4 \leq 7}\left[m_{4,7}(M) r^{4} s^{7}\right]+\sum_{7 \leq 7}\left[m_{7,7}(M) r^{7} s^{7}\right] \\
+ & \sum_{7 \leq 10}\left[m_{7,10}(M) r^{7} s^{10}\right]+\sum_{10 \leq 10}\left[m_{10,10}(M) r^{10} s^{10}\right] \\
= & m_{4,4} r^{4} s^{4}+m_{4,7} r^{4} s^{7}+m_{7,7} r^{7} s^{7}+m_{7,10} r^{7} s^{10}+m_{10,10} r^{10} s^{10} \\
= & 6 r^{4} s^{4}+(4 p+4) r^{4} s^{7}+(8 p-2) r^{7} s^{7}+(8 p-14) r^{7} s^{10}+(p-3) r^{10} s^{10}, \\
M(M, r, s)= & 6 r^{4} s^{4}+(4 p+4) r^{4} s^{7}+(8 p-2) r^{7} s^{7}+(8 p-14) r^{7} s^{10} \\
& +(p-3) r^{10} s^{10},
\end{aligned}
$$

are the required M-polynomials.

Graphical representation of M-polynomial where $p=4$ is shown in Figure 5.

Theorem 2. Let $M=L(C S(p))$ be the line graph of chain silicate network and be its M-polynomial. Then, first Zagreb index, second Zagreb index, second modified Zagreb, and general Randic, $\left(R_{\alpha}(M)\right)$, where $\alpha \in N, \quad\left(R R_{\alpha}(M)\right)$, where $\alpha \in N$ and $(S D D(M))$, obtained from M-polynomial are as follows: 
TABLe 1: Derivation for M-polynomials.

\begin{tabular}{lcc}
\hline Indices & $g\left(r_{1}, s_{1}\right)$ & Derivation from $M\left(M, r_{1}, s_{1}\right)=g\left(r_{1}, s_{1}\right)$ \\
\hline$M_{1}$ & $r_{1}+s_{1}$ & $\left.\left(D_{r 1}+D_{s 1}\right)\left(g\left(r_{1}, s_{1}\right)\right)\right|_{r_{1}=s_{1}=1}$ \\
$M_{2}$ & $r_{1} s_{1}$ & $\left.D_{r 1}\left(D_{s 1}\left(g\left(r_{1}, s_{1}\right)\right)\right)\right|_{r_{1}=s_{1}=1}$ \\
$M M_{2}$ & $1 / r_{1} s_{1}$ & $\left.S_{r 1}\left(S_{s 1}\left(g\left(r_{1}, s_{1}\right)\right)\right)\right|_{r_{1}=s_{1}=1}$ \\
$R_{\alpha}$ & $\left(r_{1} s_{1}\right)^{\alpha}$ & $\left.\left(D_{r 1}^{\alpha}\left(D_{s}^{\alpha}\right)\left(g\left(r_{1}, s_{1}\right)\right)\right)\right|_{r_{1}=s_{1}=1}$ \\
$R R_{\alpha}$ & $\left(1 / r_{1} s_{1}\right)^{\alpha}$ & $\left.\left(S_{r 1}^{\alpha}\left(S_{s 1}^{\alpha}\right)\left(g\left(r_{1}, s_{1}\right)\right)\right)\right|_{r_{1}=s_{1}=1}$ \\
SSD & $r_{1}^{2}+s_{1}^{2} / r_{1} s_{1}$ & $\left.\left(D_{r 1} S_{s 1}+D_{s 1} S_{r 1}\right)\left(g\left(r_{1}, s_{1}\right)\right)\right|_{r_{1}=s_{1}=1}$ \\
$H$ & $2 / r_{1}+s_{1}$ & $\left.2 S_{r 1} J\left(g\left(r_{1}, s_{1}\right)\right)\right|_{r_{1}=s_{1}=1}$ \\
IS & $r_{1} s_{1} / r_{1}+s_{1}$ & $\left.S_{r 1} Q_{2} J D_{r 1} D_{s 1}\left(g\left(r_{1}, s_{1}\right)\right)\right|_{r_{1}=s_{1}=1}$ \\
AZI & $\left(r_{1} s_{1} / r_{1}+s_{1}-2\right)^{3}$ & $\left.S_{r 1}^{3} J D_{r 1}^{3} D_{s 1}^{3}\left(g\left(r_{1}, s\right)\right)\right|_{r_{1}=s_{1}=1} ^{3}$ \\
\hline
\end{tabular}

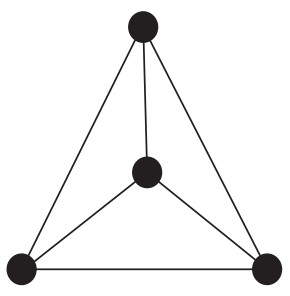

(a)

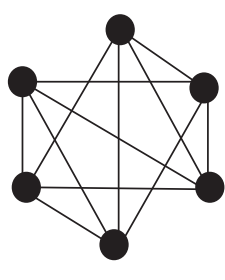

(b)

FIgURe 1: Chain silicate network CS(1) and its line graph $L(\mathrm{CS}(1))$ (left to right).

$$
\begin{aligned}
M_{1}(M)= & 312 p-234 \\
M_{2}(M)= & 1164 p-1170, \\
M M_{2}(M)= & \frac{2421}{9800}+\frac{2109 p}{4900}, \\
R_{\alpha}(M)= & 7^{\alpha}\left[4^{\alpha+1}(p+1)+7^{\alpha}(8 p-2)+10^{\alpha}(8 p-14)\right] \\
& +4^{\alpha}\left[4^{\alpha}(6)+25^{\alpha}(p-3)\right], \\
R R_{\alpha}(M)= & \frac{1}{4^{\alpha}}\left[\frac{6}{4^{\alpha}}+\frac{p-3}{25^{\alpha}}\right] \\
& +\frac{1}{7^{\alpha}}\left[\frac{(4 p+4)}{4^{\alpha}}+\frac{8 p-2}{7^{\alpha}}+\frac{8 p-14}{10^{\alpha}}\right], \\
\operatorname{SSD}(M)= & \frac{3102 p}{70}-\frac{648}{35^{2}} .
\end{aligned}
$$

TABle 2: Edges of line graph of chain silicate network.

\begin{tabular}{cccccc}
\hline Edges & $m_{4,4}$ & $m_{4,7}$ & $m_{7,7}$ & $m_{7,10}$ & $m_{10,10}$ \\
\hline$p=1$ & 12 & 0 & 0 & 0 & 0 \\
$p=2$ & 6 & 13 & 14 & 0 & 0 \\
$p=3$ & 6 & 16 & 22 & 10 & 0 \\
$p>3$ & 6 & $4 p+4$ & $8 p-2$ & $8 p-14$ & $p-3$ \\
\hline
\end{tabular}

Proof. Let $g(r, s)=M(M, r, s)$ be the M-polynomial of the line graph of chain silicate network. Then,

$$
\begin{aligned}
g(r, s)= & 6 r^{4} s^{4}+(4 p+4) r^{4} s^{7}+(8 p-2) r^{7} s^{7}+(8 p-14) r^{7} s^{10} \\
& +(p-3) r^{10} s^{10}
\end{aligned}
$$

Now, the required partial derivatives and integrals are obtained as

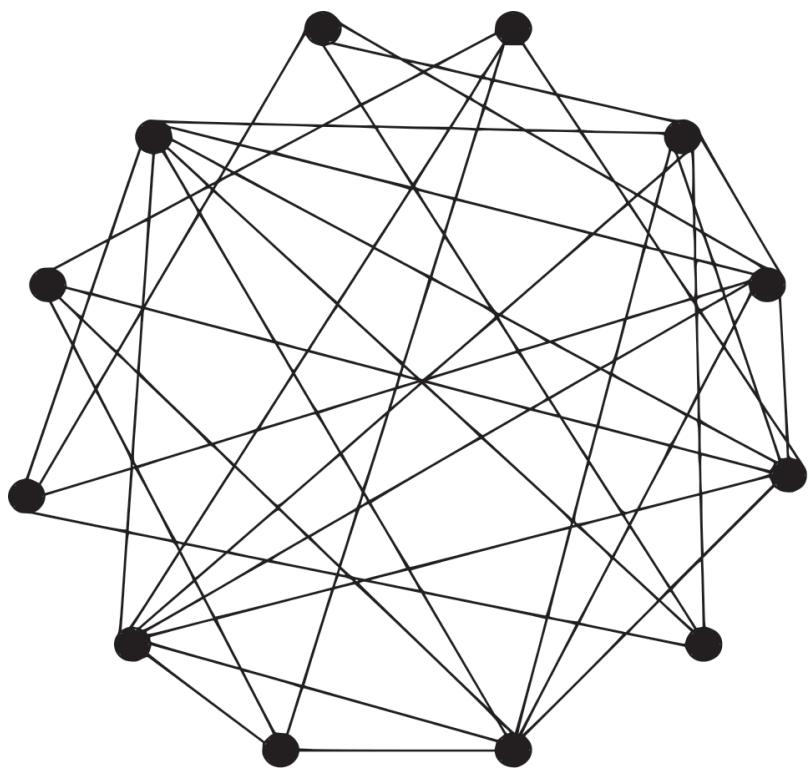

Figure 2: Graph of $L(\mathrm{CS}(2))$. 


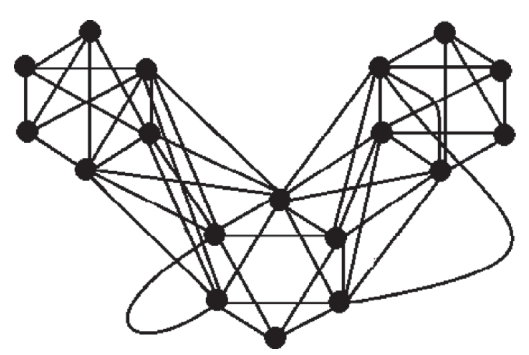

Figure 3: Graph of $L(\mathrm{CS}(3))$.

$$
\begin{aligned}
D_{r}(g(r, s))= & 24 r^{3} s^{4}+4(4 p+4) r^{3} s^{7}+7(8 p-2) r^{6} s^{7} \\
& +7(8 p-14) r^{6} s^{10}+10(p-3) r^{9} s^{10}, \\
D_{s}(g(r, s))= & 24 r^{4} s^{4}+7(4 p+4) r^{4} s^{6}+7(8 p-2) r^{7} s^{6} \\
& +10(8 p-14) r^{7} s^{9}+10(p-3) r^{10} s^{9}, \\
D_{r}\left(D_{s}((g(r, s)))\right)= & 96 r^{3} s^{3}+28(4 p+4) r^{3} s^{6}+49(8 p-2) r^{6} s^{6} \\
& +70(8 p-14) r^{6} s^{9}+100(p-3) r^{9} s^{9}, \\
S_{r}(g(r, s))= & \frac{6 r^{4} s^{4}}{4}+\frac{(4 p+4) r^{4} s^{7}}{4}+\frac{(8 p-2) r^{7} s^{7}}{7}+\frac{(8 p-14) r^{7} s^{10}}{7}+\frac{(p-3) r^{10} s^{10}}{10}, \\
S_{s}(g(r, s))= & \frac{6 r^{4} s^{4}}{4}+\frac{(4 p+4) r^{4} s^{7}}{7}+\frac{(8 p-2) r^{7} s^{7}}{7}+\frac{(8 p-14) r^{7} s^{10}}{10}+\frac{(p-3) r^{10} s^{10}}{10}, \\
S_{r} S_{s}(g(r, s))= & \frac{6 r^{4} s^{4}}{16}+\frac{(4 p+4) r^{4} s^{7}}{28}+\frac{(8 p-2) r^{7} s^{7}}{49}+\frac{(8 p-14) r^{7} s^{10}}{70}+\frac{(p-3) r^{10} s^{10}}{100}, \\
D_{s} S_{r}(g(r, s))= & 6 r^{4} s^{3}+7(p+1) r^{4} s^{6}+(8 p-2) r^{7} s^{6}+\frac{10(8 p-14) r^{7} s^{9}}{7}+(p-3) r^{10} s^{9}, \\
S_{r}^{\alpha} S_{s}^{\alpha}(g(r, s))= & \frac{6 r^{4} s^{4}}{16^{\alpha}}+\frac{(4 p+4) r^{4} s^{7}}{28^{\alpha}}+\frac{(8 p-2) r^{7} s^{7}}{49^{\alpha}}+\frac{(8 p-14) r^{7} s^{10}}{70^{\alpha}}+\frac{(p-3) r^{10} s^{10}}{100^{\alpha}} \\
D_{r} S_{s}(g(r, s))= & 6 r^{3} s^{4}+\frac{4(4 p+4) r^{3} s^{7}}{7}+(8 p-2) r^{6} s^{7}+\frac{7(8 p-14) r^{6} s^{10}}{10}+(p-3) r^{9} s^{10}, \\
D_{r}^{\alpha} D_{s}^{\alpha}(g(r, s))= & 16^{\alpha}\left(6 r^{3} s^{3}\right)+28^{\alpha}(4 p+4) r^{3} s^{6}+49^{\alpha}(8 p-2) r^{6} s^{6}
\end{aligned}
$$

Now, we obtain 


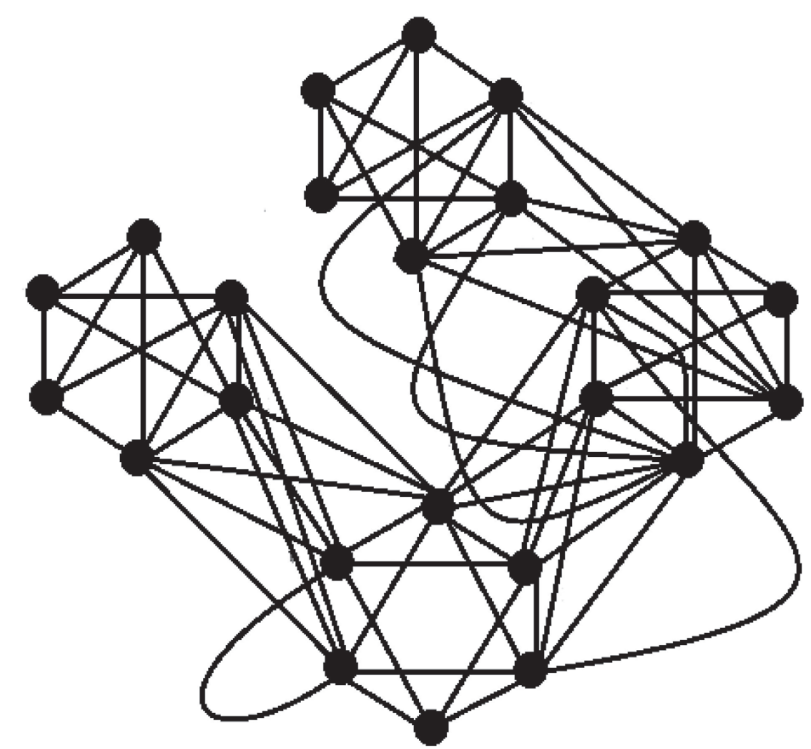

Figure 4: Graph of $L(\mathrm{CS}(4))$.

$$
\begin{aligned}
& \left.D_{r}(g(r, s))\right|_{r=s=1}=24+4(4 p+4)+7(8 n-2)+7(8 p-14)+10(p-3), \\
& \left.D_{y}(g(r, s))\right|_{r=s=1}=24+7(4 p+4)+7(8 p-2)+10(8 p-14)+10(p-3), \\
& \left.D_{r}\left(D_{s}((g(r, s)))\right)\right|_{r=s=1}=96+28(4 p+4)+49(8 p-2)+70(8 p-14)+100(p-3), \\
& \left.S_{r}(g(r, s))\right|_{r=s=1}=\frac{6}{4}+\frac{(4 p+4)}{4}+\frac{8 p-2}{7}+\frac{(8 p-14)}{7}+\frac{p-3}{10}, \\
& \left.S_{s}(g(r, s))\right|_{r=s=1}=\frac{6}{4}+\frac{4 p+4}{7}+\frac{8 p-2}{7}+\frac{8 p-14}{10}+\frac{p-3}{10} \\
& \left.S_{r} S_{s}(g(r, s))\right|_{r=s=1}=\frac{6}{16}+\frac{4 p+4}{28}+\frac{8 p-2}{49}+\frac{8 p-14}{70}+\frac{p-3}{100}, \\
& \left.D_{s} S_{r}(g(r, s))\right|_{r=s=1}=6+7\left(p+1+(8 p-2)+\frac{10(8 p-14)}{7}+(p-3)\right) \\
& \left.D_{r} S_{s}(g(r, s))\right|_{r=s=1}=6+\frac{4(4 p+4)}{7}+(8 p-2)+\frac{7(8 p-14)}{10}+(p-3) \\
& \left.D_{r}^{\alpha} D_{s}^{\alpha}(g(r, s))\right|_{r=s=1}=16^{\alpha}(6)+28^{\alpha}(4 p+4)+49^{\alpha}(8 p-2)+70^{\alpha}(8 p-14)+100^{\alpha}(p-3), \\
& \left.S_{r}^{\alpha} S_{s}^{\alpha}(g(r, s))\right|_{r=s=1}=\frac{6}{16^{\alpha}}+\frac{4 p+4}{28^{\alpha}}+\frac{(8 p-2)}{49^{\alpha}}+\frac{(8 p-14)}{70^{\alpha}}+\frac{(p-3)}{100^{\alpha}} .
\end{aligned}
$$

Consequently, 


$$
\begin{aligned}
M_{1}(M)= & \left.\left(D_{r}+D_{s}\right)(g(r, s))\right|_{r=s=1} \\
= & \left.D_{r}(g(r, s))\right|_{r=s=1}+\left.D_{s}(g(r, s))\right|_{r=s=1}=312 p-234 \\
M_{2}(M)= & \left.\left(D_{r} D_{s}\right)(g(r, s))\right|_{r=s=1} \\
= & \left.D_{r}\left(D_{s}(g(r, s))\right)\right|_{r=s=1}=1164 p-1170, \\
M M_{2}(M)= & \left.\left(S_{r} S_{s}\right)(g(r, s))\right|_{r=s=1} \\
= & \left.S_{r}\left(S_{s}(g(r, s))\right)\right|_{r=s=1} \\
= & \frac{2421}{9800}+\frac{2109 p}{4900}, \\
\operatorname{SSD}_{\alpha}(M)= & \left.\left(D_{r}^{\alpha} D_{s}^{\alpha}\right)(g(r, s))\right|_{r=s=1} \\
= & \left.\left(D_{r}^{\alpha}\left(S_{s}^{\alpha}\right)(g(r, s))\right)\right|_{r=s=1} \\
& +4_{r}^{\alpha}\left[\left.S_{s}(g(r, s))\right|_{r=s=1}+\left.D_{s} S_{r}(g(r, s))\right|_{r=s=1}+25^{\alpha}(p-3)\right], \\
= & \left.\left(S_{r}^{\alpha} S_{s}^{\alpha}\right)(g(r, s))\right|_{r=s=1} \\
= & \left.\left(S_{r}^{\alpha}\left(S_{s}^{\alpha}\right)(g(r, s))\right)\right|_{r=s=1}\left[4^{\alpha+1}(p+1)+7^{\alpha}(8 p-2)+10^{\alpha}(8 p-14)\right] \\
= & \frac{1}{4^{\alpha}}\left[\frac{6}{4^{\alpha}}+\frac{p-3}{25^{\alpha}}\right]+\frac{1}{7^{\alpha}}\left[\frac{(4 p+4)}{4^{\alpha}}+\frac{8 p-2}{7^{\alpha}}+\frac{8 p-14}{10^{\alpha}}\right] \\
= & \\
= & \\
= &
\end{aligned}
$$

Theorem 3. Let $M=L(C S(p))$ be the line graph of chain silicate network and

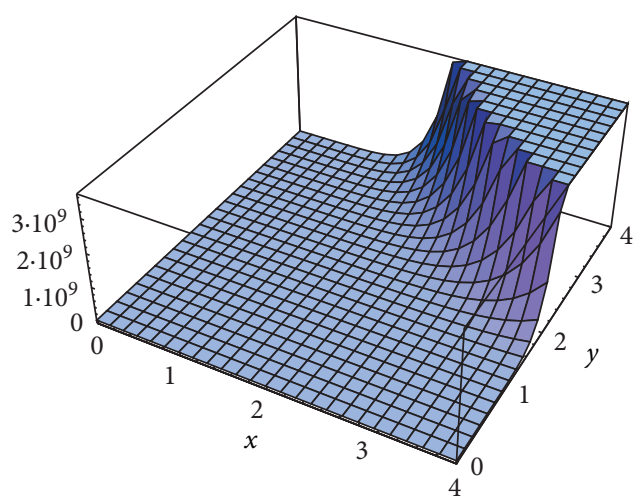

FIGURE 5: Graphical representation of M-polynomial where $p=4$.

$$
\begin{aligned}
M(M, r, s)= & 6 r^{4} s^{4}+(4 p+4) r^{4} s^{7}+(8 p-2) r^{7} s^{7}+(8 p-14) r^{7} s^{10} \\
& +(p-3) r^{10} s^{10}
\end{aligned}
$$

be $M=L(C S(p))$ M-polynomial. Then, $(H(M))$, (IS $(M))$, and $(A Z I(M))$ obtained from M-polynomial are as follows:

$$
\begin{aligned}
H(M) & =\frac{-36}{6545}+\frac{38109 p}{13090} \\
\operatorname{IS}(M) & =\frac{3(-1924539+2432060 p)}{108680}, \\
\operatorname{AZI}(M) & =\frac{-42903883+38482476 p}{23328}
\end{aligned}
$$

Proof. Let $g(r, s)=M(M, r, s)$ be a M-polynomial of the line graph of chain silicate network. Then,

$$
\begin{aligned}
g(r, s)= & 6 r^{4} s^{4}+(4 p+4) r^{4} s^{7}+(8 p-2) r^{7} s^{7} \\
& +(8 p-14) r^{7} s^{10}+(p-3) r^{10} s^{10} .
\end{aligned}
$$

Now, the required expressions are obtained as 


$$
\begin{aligned}
& J(g(r, s))=6 r^{8}+(4 p+4) r^{11}+(8 p-2) r^{14}+(8 p-14) r^{17}+(p-3) r^{20}, \\
& S_{r}(J(g(r, s)))=\frac{6 r^{8}}{8}+\frac{(4 p+4) r^{11}}{11}+\frac{(8 p-2) r^{14}}{14}+\frac{(8 p-14) r^{17}}{17}+\frac{(p-3) r^{20}}{20} \\
& J\left(D_{r}\left(D_{s}((g(r, s)))\right)\right)=96 r^{6}+28(4 p+4) r^{9}+49(8 p-2) r^{12}+70(8 p-14) r^{15}+100(p-3) r^{18}, \\
& Q_{2} J\left(D_{r}\left(D_{s}((g(r, s)))\right)\right)=96 r^{8}+28(4 p+4) r^{11}+49(8 p-2) r^{14}+70(8 p-14) r^{17}+100(p-3) r^{20} \text {, } \\
& S_{r} Q_{2} J\left(D_{r}\left(D_{s}((g(r, s)))\right)\right)=\frac{96 r^{8}}{8}+\frac{28(4 p+4) r^{11}}{11}+\frac{49(8 p-2) r^{14}}{14}+\frac{70(8 p-14) r^{17}}{17}+\frac{100(p-3) r^{20}}{20} \\
& D_{r}^{3} D_{s}^{3}(g(r, s))=16^{3}\left(6 r^{3} s^{3}\right)+28^{3}(4 p+4) r^{3} s^{6}+49^{3}(8 p-2) r^{6} s^{6}+70^{3}(8 p-14) r^{6} s^{9}+100^{3}(p-3) r^{9} s^{9}, \\
& J D_{r}^{3} D_{s}^{3}(g(r, s))=16^{3}\left(6 r^{6}\right)+28^{3}(4 p+4) r^{9}+49^{3}(8 p-2) r^{12}+70^{3}(8 p-14) r^{15}+100^{3}(p-3) r^{18}, \\
& S_{r}^{3} J D_{r}^{3} D_{s}^{3}(g(r, s))=\left(\frac{16}{6}\right)^{3} 6 r^{6}+\left(\frac{28}{9}\right)^{3}(4 p+4) r^{9}+\left(\frac{49}{12}\right)^{3}(8 p-2) r^{12}+\left(\frac{70}{15}\right)^{3}(8 p-14) r^{15} \\
& +\left(\frac{100}{18}\right)^{3}(p-3) r^{18}
\end{aligned}
$$

Now, we obtain

$$
\begin{aligned}
\left.J(g(r, s))\right|_{r=s=1} & =6+(4 p+4)+(8 n-2)+(8 p-14)+(p-3), \\
\left.S_{r}(J(g(r, s)))\right|_{r=s=1} & =\frac{6}{8}+\frac{4 p+4}{11}+\frac{8 p-2}{14}+\frac{8 p-14}{17}+\frac{p-3}{20}, \\
\left.J\left(D_{r}\left(D_{s}((g(r, s)))\right)\right)\right|_{r=s=1} & =96+28(4 p+4)+49(8 p-2)+70(8 p-14)+100(p-3), \\
\left.Q_{2} J\left(D_{r}\left(D_{s}((g(r, s)))\right)\right)\right|_{r=s=1} & =96+28(4 p+4)+49(8 p-2)+70(8 p-14)+100(p-3), \\
\left.S_{r} Q_{2} J\left(D_{r}\left(D_{s}((g(r, s)))\right)\right)\right|_{r=s=1} & =\frac{96}{8}+\frac{28(4 p+4)}{11}+\frac{49(8 p-2)}{14}+\frac{70(8 p-14)}{17}+\frac{100(p-3)}{20}, \\
\left.D_{r}^{3} D_{s}^{3}(g(r, s))\right|_{r=s=1} & =16^{3}(6)+28^{3}(4 p+4)+49^{3}(8 p-2)+70^{3}(8 p-14)+100^{3}(p-3), \\
\left.J D_{r}^{3} D_{s}^{3}(g(r, s))\right|_{r=s=1} & =16^{3}(6)+28^{3}(4 p+4)+49^{3}(8 p-2)+70^{3}(8 p-14)+100^{3}(p-3), \\
\left.S_{x}^{3} J D_{x}^{3} D_{y}^{3}(g(r, s))\right|_{r=s=1} & =\left(\frac{16}{6}\right)^{3} 6+\left(\frac{28}{9}\right)^{3}(4 p+4)+\left(\frac{49}{12}\right)^{3}(8 p-2)+\left(\frac{70}{15}\right)^{3}(8 p-14)+\left(\frac{100}{18}\right)^{3}(p-3) .
\end{aligned}
$$

\section{Consequently,}




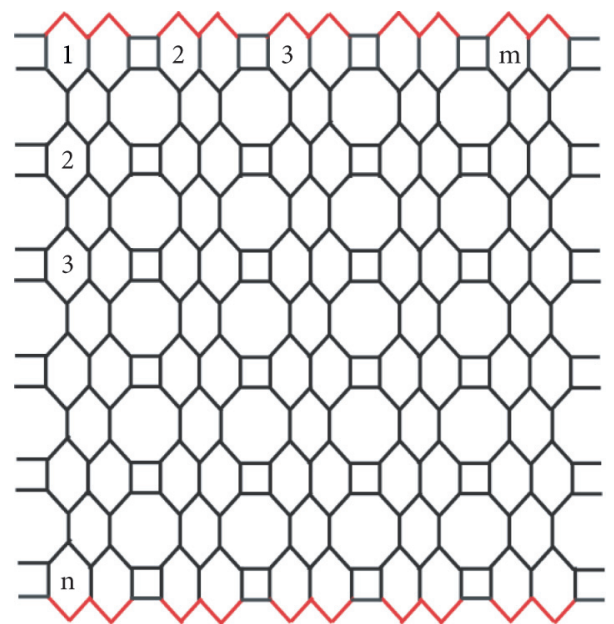

Figure 6: Graph of NPHX $[x, y]$.

$$
\begin{aligned}
& H(M)=\left.2 S_{r}(J(g(r, s)))\right|_{r=s=1} \\
& =2\left(\frac{6}{8}+\frac{4 p+4}{11}+\frac{8 p-2}{14}+\frac{8 p-14}{17}+\frac{p-3}{20}\right) \\
& =\frac{-36}{6545}+\frac{38109 p}{13090} \\
& \operatorname{IS}(M)=\left.S_{r} Q_{2} J\left(D_{r}\left(D_{s}((g(r, s)))\right)\right)\right|_{r=s=1} \\
& =\frac{96}{8}+\frac{28(4 p+4)}{11}+\frac{49(8 p-2)}{14} \\
& +\frac{70(8 p-14)}{17}+\frac{100(p-3)}{20} \\
& =\frac{3(-1924539+2432060 p)}{108680} \text {, } \\
& \operatorname{AZI}(M)=\left.S_{r}^{3} J D_{r}^{3} D_{s}^{3}(g(r, s))\right|_{r=s=1} \\
& =\left(\frac{16}{6}\right)^{3} 6+\left(\frac{28}{9}\right)^{3}(4 p+4)+\left(\frac{49}{12}\right)^{3}(8 p-2) \\
& +\left(\frac{70}{15}\right)^{3}(8 p-14)+\left(\frac{100}{18}\right)^{3}(p-3) \\
& =\frac{-42903883+38482476 p}{23328} \text {. }
\end{aligned}
$$

3.1. H-Naphtalenic Nanotubes. In line graph of H-naphtalenic nanotubes $L(\mathrm{NPHX}[x, y])$, there are two types of vertices having degrees 3 and 4 and there are three partitions of edges such as $m_{3,3}, m_{3,4}$, and $m_{4,4} . m_{3,3}, m_{3,4}$, and $m_{4,4}$ means there are three edges between the vertices of degrees $3,3,3,4$, and 4, 4. Graphs of $\mathrm{H}$-naphtalenic nanotubes and its line graphs for $x, y=1$, are shown in Figures 6 to 8. Total edges and vertices of line graph of $\mathrm{H}$-naphtalenic nanotubes
$(L(\operatorname{NPHX}[x, y]))$ ([21] for detail) are $30 x y-8 x$ and $15 x y-2 x$, respectively.

Theorem 4. Let $N=L(N P H X[x, y])$ be the line graph of $H$-naphtalenic nanotubes. Then, the M-polynomial of $N$ is

$$
M(N, r, s)=6 x r^{3} s^{3}+12 x r^{3} s^{4}+(30 x y-26 x) r^{4} s^{4} .
$$

Proof. From Figures 6 to 8, we noted that there are two types of vertices and three types of edges, detail given below:

$$
\begin{aligned}
& m_{3,3}, d(k)=d(l)=3, \\
& m_{3,4}, d(k)=3, d(l)=4, \\
& m_{4,4}, d(k)=4, d(l)=4 .
\end{aligned}
$$

We conclude that

$$
\begin{aligned}
& |V(L(\operatorname{NPHX}[x, y]))|=15 x y-2 x, \\
& |E(L(\operatorname{NPHX}[x, y]))|=30 x y-8 x .
\end{aligned}
$$

M-Polynomial of $N$ is

$$
\begin{aligned}
M(N, r, s)= & \sum_{p \leq q}\left[m_{p, q}(N) r^{p} s^{q}\right] \\
= & \sum_{3 \leq 3}\left[m_{3,3}(N) r^{3} s^{3}\right]+\sum_{3 \leq 4}\left[m_{3,4}(N) r^{3} s^{4}\right] \\
& +\sum_{4 \leq 4}\left[m_{4,4}(N) r^{4} s^{4}\right] \\
= & m_{3,3} r^{3} s^{3}+m_{3,4} r^{3} s^{4}+m_{4,4} r^{4} s^{4} \\
= & 6 x r^{3} s^{3}+12 x r^{3} s^{4}+(30 x y-26 x) r^{4} s^{4} .
\end{aligned}
$$

Graphical representation of M-polynomial of line graph of H-naphtalenic nanotubes $(\operatorname{L}(\operatorname{NPHX}[x, y]))$ is shown in Figure 9.

Theorem 5. Let $N=L(N P H X[x, y])$ be the line graph of $H$-naphtalenic nanotubes and M-polynomial of $N$ is 


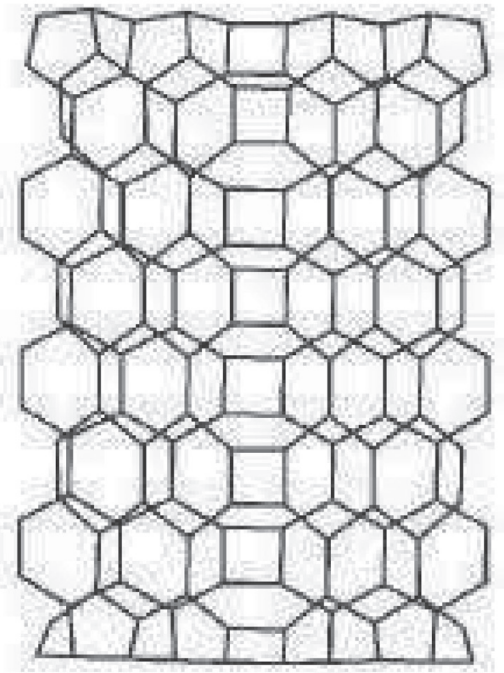

FIGURE 7: Graph of NPHX $[x, y]$.

$M(N, r, s)=)=6 x r^{3} s^{3}+12 x r^{3} s^{4}+(30 x y-26 x) r^{4} s^{4}$,

then

$$
\begin{aligned}
M_{1}(L(\operatorname{NPHX}[x, y]))= & -88 x+240 x y, \\
M_{2}(L(\operatorname{NPHX}[x, y]))= & 218 x+480 x y, \\
M M_{2}(L(\operatorname{NPHX}[x, y]))= & \frac{1}{24}(x+45 x y), \\
R_{\alpha}(L(\operatorname{NPHX}[x, y]))= & (2) 3^{1+2 \alpha} x+12^{1+\alpha} x \\
& +16^{\alpha}(-26 x+30 x y), \\
R R_{\alpha}(L(\operatorname{NPHX}[x, y]))= & 3^{1-2 \alpha}(2 x)+(12)^{1-\alpha} \\
& +16^{-\alpha}(30 x y-26 x), \\
\operatorname{IS}(L(\operatorname{NPHX}[x, y]))= & \frac{-157 x}{7}+60 x y, \\
H(L(\operatorname{NPHX}[x, y]))= & \frac{-15}{14}(x-7 x y), \\
\operatorname{SDD}(L(\operatorname{NPHX}[x, y]))= & -15(x-4 x y), \\
\operatorname{AZI}(L(\operatorname{NPHX}[x, y]))= & \frac{-27950971 x}{108000}+\frac{5120 x y}{9} .
\end{aligned}
$$

Proof. Same as above.

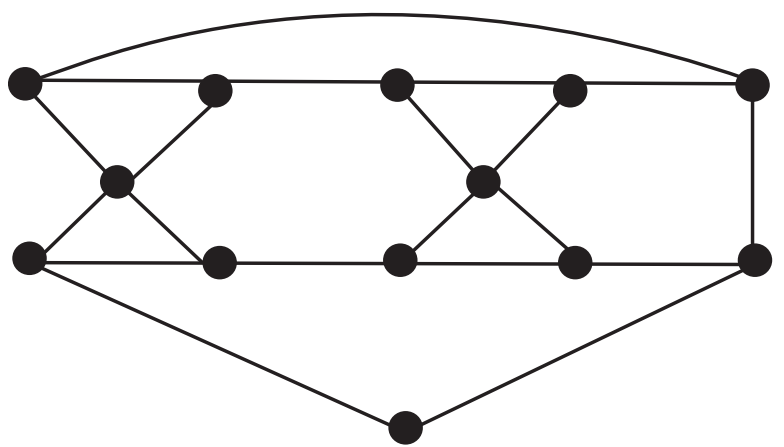

Figure 8: Graph of $L($ NPHX $[1,1])$.

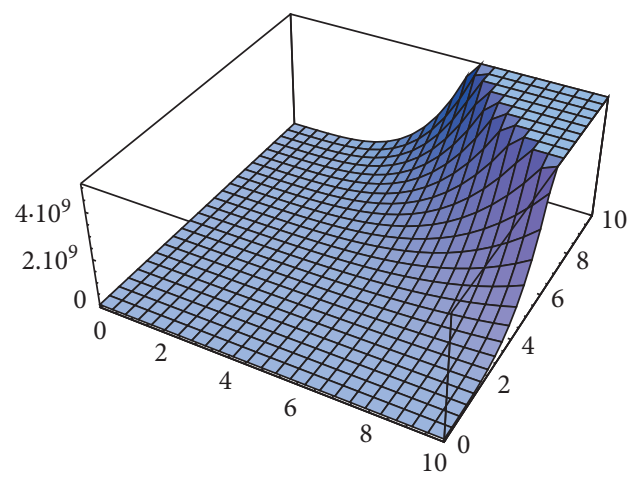

Figure 9: M-Polynomial of line graph of $\mathrm{H}$-naphtalenic nanotubes $(L(\operatorname{NPHX}[x, y]))$.

\section{Conclusion}

In this paper, we derived the results of M-polynomial for line graph of chain silicate network. M-polynomial gives the source of information about the closed form of topological indices based on degrees of molecular graph $M$. These results can play an essential role to determine the properties and uses of this network in industries, electronics, and pharmacies.

\section{Data Availability}

No data were used to support this study.

\section{Conflicts of Interest}

The authors declare no conflicts of interest.

\section{Authors' Contributions}

All authors contributed equally in writing this paper. All authors read and approved the final manuscript. 


\section{Acknowledgments}

The work was supported by the Higher Education Commission of Pakistan.

\section{References}

[1] V. Alamian, A. Bahrami, and B. Edalatzadeh, "PI polynomial of V-phenylenic nanotubes and nanotori," International Journal of Molecular Sciences, vol. 9, no. 3, pp. 229-234, 2008.

[2] G. Fath-Tabar, "Old and new zagreb indices of graphs," MATCH Communications in Mathematical and in Computer Chemistry, vol. 65, pp. 79-84, 2011.

[3] B. Furtula, A. Graovac, and D. Vukičević, "Augmented zagreb index," Journal of Mathematical Chemistry, vol. 48, no. 2, pp. 370-380, 2010 .

[4] M. Ghorbani, H. Mesgarani, and S. Shakeraneh, "Computing GA index and ABC index of V-phenylenic nanotube, Optoelectron," Advanced Materials - Rapid Communications, vol. 5, pp. 324-326, 2011.

[5] E. Deutsch and S. Klavzar, "M-Polynomial and degree-based topological indices, Iran,” J. Math. Chem.vol. 6, pp. 93-102, 2015.

[6] N. Trinajstic, S. Nikolic, A. Milicevic, and I. Gutman, "On zagreb indices,” Kemind Indices, vol. 59, pp. 577-589, 2010.

[7] A. N. A. Koam, A. Ahmad, and M. F. Nadeem, "Comparative study of valency-based topological descriptor for hexagon star network," Computer Systems Science and Engineering, vol. 36, no. 2, pp. 293-306, 2021.

[8] A. Ahmad, "Topological properties of Sodium chloride," UPB Scientific Bulletin, Series B, vol. 82, no. 1, pp. 35-46, 2020.

[9] A. Ahmad, "On the degree based topological indices of Benzene ring embedded in the P-type-surface in 2D network," Hacettepe Journal of Mathematics and Statistics, vol. 47, no. 1, pp. 9-18, 2018.

[10] M. Randic, "Characterization of molecular branching," Journal of the American Chemical Society, vol. 97, no. 23, pp. 6609-6615, 1975.

[11] B. Bollobas and P. Erdos, "Graphs of extremal weights," Ars Combinatoria, vol. 50, pp. 225-233, 1998.

[12] D. Amic, D. Beslo, B. Lucic, S. Nikolic, and N. Trinajstic, "The vertex-connectivity index revisited," Journal of Chemical Information and Computer Sciences, vol. 38, pp. 819-822, 1998.

[13] G. Caporossi, I. Gutman, P. Hansen, and L. Pavlović, "Graphs with maximum connectivity index," Computational Biology and Chemistry, vol. 27, no. 1, pp. 85-90, 2003.

[14] A. Bahrami and J. Yazdani, "Vertex PI index of V-phenylenic nanotubes and nanotori," Digest Journal of Nanomaterials and Biostructures, vol. 4, pp. 141-144, 2009.

[15] M. Munir, W. Nazeer, S. Rafique, and S. Kang, "M-polynomial and related topological indices of Nanostar dendrimers," Symmetry, vol. 8, no. 9, p. 97, 2016.

[16] M. Munir, W. Nazeer, S. Rafique, and S. Kang, "M-polynomial and degree-based topological indices of polyhex nanotubes," Symmetry, vol. 8, no. 12, p. 149, 2016.

[17] Y. C. Kwun, M. Munir, W. Nazeer, S. Rafique, and S. M. Kang, "M-Polynomials and topological indices of V-phenylenic nanotubes and nanotori," Scientific Reports, vol. 7, pp. 87-56, 2017.

[18] A. Ahmad, R. Hasni, K. Elahi, and M. A. Asim, "Polynomials of degree-based indices for swapped networks modeled by optical transpose interconnection system," IEEE Access, vol. 8, pp. 214293-214299, 2020.
[19] A. N. A. Koam and A. Ahmad, "Polynomials of degree-based indices for three dimensional mesh network," Computers, Materials and Continua, vol. 65, no. 2, pp. 1271-1282, 2020.

[20] M. U. Rehman, M. Javaid, and J. Cao, "Topological indices of rhombus type silicate and oxide networks," Canadian Journal of Chemistry, vol. 95, pp. 134-143, 2017.

[21] H. Sakander and M. Imran, "On degree based topological indices of certain nanotubes," Jounal of Computational and Theoretical Nanoscience, vol. 12, no. 8, 2015. 